\title{
Leptogenesis in the minimal supersymmetric triplet seesaw model
}

\author{
Giancarlo D'Ambrosio, ${ }^{1}$ Thomas Hambye, ${ }^{2}$ Andi Hektor, ${ }^{3}$ Martti Raidal, ${ }^{3}$ and Anna Rossi ${ }^{4}$ \\ ${ }^{1}$ INFN, Sezione di Napoli and Dipartimento di Scienze Fisiche, Università di Napoli, I-80126 Naples, Italy \\ ${ }^{2}$ Theoretical Physics Subdept, University of Oxford, 1 Keble Road, Oxford OX13NP, UK \\ ${ }^{3}$ National Institute of Chemical Physics and Biophysics, Ravala 10, Tallinn 10143, Estonia \\ ${ }^{4}$ Dipartimento di Fisica "G. Galilei", Università di Padova and INFN, Sezione di Padova, I-35131 Padua, Italy
}

(Dated: August 10, 2018)

\begin{abstract}
In the supersymmetric triplet (type-II) seesaw model, in which a single $S U(2)_{L}$-triplet couples to leptons, the high-energy neutrino flavour structure can be directly determined from the low-energy neutrino data. We show that even with such a minimal triplet content, leptogenesis can be naturally accommodated thanks to the resonant interference between superpotential and soft supersymmetry breaking terms.
\end{abstract}

1. Introduction. Non-zero neutrino masses and mixing angles provide the only convincing evidence [1] of physics beyond the standard model we have today. The seesaw mechanism can be regarded as a paradigm to understand these neutrino properties. According to this mechanism at the decoupling of some heavy states with mass larger than the electroweak scale, and coupled to $S U(2)_{L}$ lepton and Higgs doublets, the $d=5$ operator $\frac{\mathcal{K}}{M} L L H H$ is generated, where $M$ is a typical scale at which the lepton number is violated. Upon breaking of $S U(2)_{L} \times U(1)_{Y}$, by $\langle H\rangle=v$, Majorana masses for the neutrinos are induced, $m_{\nu}=\frac{v^{2}}{M} \mathcal{K}$. In the basis in which the charged-lepton mass matrix is diagonal, all the lowenergy flavour structure is contained in the matrix $\mathcal{K}$, i.e. in the neutrino mass matrix. For neutrino masses in the range $\sim\left(10^{-1}-1\right) \mathrm{eV}$, the overall scale $M$ can be inferred, $\mathcal{K}^{-1} M \sim 10^{14} \mathrm{GeV}$.

The most popular realizations of the seesaw mechanism are the singlet seesaw 2] (or type-I) in which three singlet fermions $N$ are exchanged, and the triplet [3, 4] seesaw [4] (or type-II) in which a $S U(2)_{L}$ triplet scalar $T$ with non-zero hypercharge is exchanged ${ }^{1}$. The two realizations differ in an important aspect. Namely, the singlet seesaw has two sources of flavour structure, the Yukawa coupling matrix $Y_{N}$ of the singlets $N$ with the $S U(2)_{L}$ leptons, and the mass matrix $M_{N}$ of the states $N$, which combine to give $\mathcal{K} / M=Y_{N}^{T} M_{N}^{-1} Y_{N}$. The triplet seesaw scenario has only one source of flavour, i.e. the symmetric Yukawa matrix $Y_{T}$ of the triplet $T$ couplings with the leptons, and so $\mathcal{K} / M=\lambda_{2} Y_{T} / M_{T}$, where $M_{T}$ is the triplet mass and $\lambda_{2}$ is a dimensionless ('unflavoured') Higgs coupling. This implies that in the type-I seesaw case the high-energy quantities, $Y_{N}$ and $M_{N}$ which con-

\footnotetext{
${ }^{1}$ In the supersymmetric scenario, which we assume in this paper from the very beginning, the anomaly cancellation requires minimally a pair of triplets $T, \bar{T}$ with opposite $U(1)_{Y}$ quantum numbers. However, only one triplet $T$ couples to leptons.
}

tain 18 parameters ${ }^{2}$ cannot be univocally related to the 9 low-energy neutrino parameters, encoded in the symmetric neutrino mass matrix $m_{\nu}$. Instead in the type-II case the high-energy quantity $Y_{T}$, which contains 9 parameters can be matched to the flavour structure of the matrix $m_{\nu}[\underline{4},[\underline{6}]$. What is left undetermined is the overall mass scale $M_{T} / \lambda_{2}$.

As both the seesaw scenarios involve a high-energy scale where the lepton number $L$ is not conserved, either $M_{N}$ or $M_{T}$, they also offer a possibility to generate the observed baryon asymmetry of the Universe via outof-equilibrium decays of these heavy states. In the type-I seesaw model leptogenesis [7] occurs naturally (both in its supersymmetric as well as non-supersymmetric versions) since there are three heavy singlet neutrinos. However, the situation is more involved in the type-II seesaw case. The minimal triplet seesaw with one-pair $T, \bar{T}$ (or with one triplet $T$ in the non-supersymmetric case) does contain sources of $L$ and $\mathrm{CP}$ violation at high scale, but it cannot effectively induce leptogenesis [8, 9]. Successful leptogenesis has been achieved by adding one more vector-like $T^{\prime}, \bar{T}^{\prime}$ pair [8, [9], and therefore one more Yukawa matrix $Y_{T^{\prime}}$. In such a case 9 more flavour parameters will be added, and the direct connection between the high-energy flavour structure and the lowenergy one is lost. Alternatively, one can consider the hybrid type-I plus type-II model (motivated by left-right and $\mathrm{SO}(10)$ models) where a single triplet couples to leptons [10, 11, 12]. In this case, successful leptogenesis can be obtained in agreement with neutrino data from the interplay of type-I and type-II contributions [1], though the above flavour connection is also lost.

In this paper we show that leptogenesis turns out to be in fact possible in the minimal supersymmetric triplet seesaw scenario with only one pair of triplets $T, \bar{T}$. Nonvanishing interfering amplitudes emerge due to soft su-

\footnotetext{
${ }^{2}$ Predictive seesaw models, with two $N$ 's are also viable [5].
} 
persymmetry breaking mass parameters (not considered in [9]), and resonant "soft" leptogenesis [13, 14] occurs ${ }^{3}$. As a result the observed $B-L$ asymmetry can be generated due to finite temperature effects 18 .

This paper is organized as follows. In Section 2 we introduce the relevant superpotential and Lagrangian with the soft-breaking terms to describe the minimal triplet seesaw framework. In Section 3 the lepton asymmetry is derived including the thermal corrections, and hence the baryon asymmetry is obtained. We discuss our results in Section 4, give a few general remarks in Section 5 and conclude in Section 6.

2. The triplet-seesaw scenario. The supersymmetric triplet seesaw model is described by the following superpotential

$$
W=\frac{1}{\sqrt{2}}\left(Y_{T}^{i j} L_{i} T L_{j}+\lambda_{1} H_{1} T H_{1}+\lambda_{2} H_{2} \bar{T} H_{2}\right)+M_{T} T \bar{T},
$$

where $i, j=e, \mu, \tau$ are flavour indices, $T=\left(T^{0}, T^{+}, T^{++}\right)$, $\bar{T}=\left(\bar{T}^{0}, \bar{T}^{-}, \bar{T}^{--}\right)$are in a vector-like $S U(2)_{L} \times U(1)_{Y}$ representation, $T \sim(3,1)$ and $\bar{T} \sim(3,-1), L_{i}$ are the lepton $S U(2)_{L}$ doublets, and $H_{1}\left(H_{2}\right)$ is the Higgs doublet with hypercharge $-1 / 2(1 / 2)$. In eq. (11), the symmetric matrix $Y_{T}$ contains 6 real parameters plus 3 phases, while the parameters $\lambda_{2}$ and $M_{T}$ can be taken to be real, and $\lambda_{1}$ is in general complex. We observe that if, for example, we assign the lepton number $L=0$ to the triplets $T, \bar{T}$, the $Y_{T}$-couplings explicitly break $L$, while the $\lambda_{1}, \lambda_{2}$ couplings conserve $L$. By integrating out the triplet states at the scale $M_{T}$, the $d=5 L$-violating operator is generated which after the electroweak symmetry breaking induces the neutrino mass matrix

$$
m_{\nu}^{i j}=Y_{T}^{i j} \lambda_{2} \frac{v_{2}^{2}}{M_{T}} .
$$

This clearly shows that the flavour structure of neutrino mass matrix is directly linked to that of the matrix $Y_{T}$.

In the supersymmetric limit the states $T$ and $\bar{T}$ are degenerate with mass $M_{T}$. The necessary mass splitting and $\mathrm{CP}$-violation to induce resonant leptogenesis is provided by the soft supersymmetry breaking (SSB) terms. The relevant ones are:

$$
\begin{aligned}
-\mathcal{L}_{\text {soft }}= & {\left[\frac{1}{\sqrt{2}}\left(A_{T}^{i j} \tilde{L}_{i} T \tilde{L}_{j}+A_{1} H_{1} T H_{1}+A_{2} H_{2} \bar{T} H_{2}\right)\right.} \\
& \left.+B M_{T} T \bar{T}+\text { h.c. }\right]+\tilde{m}_{T}^{2} T^{\dagger} T+\tilde{m}_{\bar{T}}^{2} \bar{T}^{\dagger} \bar{T},(3)
\end{aligned}
$$

where a standard notation has been adopted. In eq. (3), the matrix $A_{T}$ has 6 real parameters plus 3 phases, and

\footnotetext{
${ }^{3}$ Other scenarios of (non-resonant) leptogenesis induced by soft supersymmetry breaking terms can be found in [15, 16]. See also [17.
}

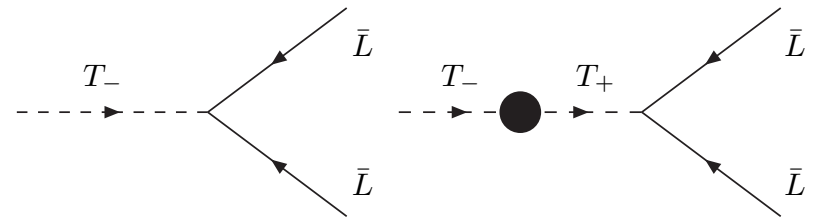

FIG. 1: Interfering $T_{-}$decay amplitudes for the fermionic final states. Analogous diagrams exist for bosonic final states. The two-point function $\Pi_{+-}$is denoted by a blob.

the mass parameters $A_{1}, A_{2}$ and $\left(B M_{T}\right)$ are in general complex. In the following, we set to zero $\arg \left(B M_{T}\right)$ without loss of generality. The $B$-term in eq. (3) removes the mass degeneracy, $M_{ \pm}^{2}=M_{T}^{2} \pm B M_{T}$ (where the SSB terms $\tilde{m}_{T(\bar{T})}^{2} \ll M_{T}^{2}$ have been neglected), between the mass eigenstates $T_{ \pm}$which are given by ${ }^{4}$

$$
T_{ \pm}=\frac{1}{\sqrt{2}}\left(T \pm \bar{T}^{*}\right) .
$$

In the $T_{ \pm}$-basis the relevant Lagrangian interactions read as:

$$
\begin{aligned}
-\mathcal{L}= & \frac{1}{\sqrt{2}}\left(Y_{T}^{i j} L_{i} \frac{T_{+}+T_{-}}{\sqrt{2}} L_{j}+\lambda_{1} \tilde{H}_{1} \frac{T_{+}+T_{-}}{\sqrt{2}} \tilde{H}_{1}\right. \\
& +\lambda_{2} \tilde{H}_{2} \frac{T_{+}^{*}-T_{-}^{*}}{\sqrt{2}} \tilde{H}_{2}+M_{T} Y_{T}^{i j} \tilde{L}_{i} \frac{T_{+}-T_{-}}{\sqrt{2}} \tilde{L}_{j} \\
& +M_{T} \lambda_{1} H_{1} \frac{T_{+}-T_{-}}{\sqrt{2}} H_{1}+M_{T} \lambda_{2} H_{2} \frac{T_{+}^{*}+T_{-}^{*}}{\sqrt{2}} H_{2} \\
& +A_{T}^{i j} \tilde{L}_{i} \frac{T_{+}+T_{-}}{\sqrt{2}} \tilde{L}_{j}+A_{1} H_{1} \frac{T_{+}+T_{-}}{\sqrt{2}} H_{1} \\
& \left.+A_{2} H_{2} \frac{T_{+}^{*}-T_{-}^{*}}{\sqrt{2}} H_{2}\right)+ \text { h.c. }
\end{aligned}
$$

3. The lepton asymmetry. To take into account the resonant phenomenon of CP-violation due to the mixing of (almost) degenerate states, as $T_{ \pm}$are, we use an effective field-theory approach of resummed propagators for unstable (mass eigenstate) particles, as described in ref. [19]. The decay amplitude $\widehat{S}_{-}^{f}$ of the unstable external state $T_{-}$into a final state $\bar{f}$ is described by a superposition of amplitudes $S_{ \pm}^{f}$ with stable external states. Adding the contributions shown in Fig. 1] we obtain

$$
\widehat{S}_{-}^{f}\left(T_{-} \rightarrow \bar{f}\right)=S_{-}^{f}-S_{+}^{f} \frac{i \Pi_{+-}}{M_{-}^{2}-M_{+}^{2}+i \Pi_{++}},
$$

where $\Pi_{\alpha \beta}\left(p^{2}\right)$ are the absorptive parts of the two-point functions for $\alpha, \beta=+,-$. The amplitude for the decay

\footnotetext{
${ }^{4}$ We have adopted the representation for $T$ and $\bar{T}$ as given in 6 . Therefore, in the r.h.s. of eq. (4) the term $\bar{T}^{*}$ is understood as $i \sigma_{2} \bar{T}^{*} i \sigma_{2}$, where $\sigma_{2}$ is the complex Pauli matrix.
} 
into the conjugate final state is obtained by the replacements $S_{ \pm} \rightarrow S_{ \pm}^{*}, \Pi_{+-} \rightarrow \Pi_{+-}^{*}$. From the amplitudes (6) the corresponding widths can be obtained. The relevant $L$ violating decays are $T_{ \pm} \rightarrow \bar{L} \bar{L}, \tilde{L}^{*} \tilde{L}^{*}$ with $\Delta L=-2$ and $T_{ \pm}^{*} \rightarrow L L, \tilde{L} \tilde{L}$ with $\Delta L=2$. The lepton asymmetry is obtained by calculating the $\Delta L$ produced on average each time any component of $T_{ \pm}$or $T_{ \pm}^{*}$ decays. Therefore, for $T_{-}$and $T_{-}^{*}$ (and similarly for $T_{+}$and $T_{+}^{*}$, by interchanging + and - ) this is given by the following $\mathrm{CP}$ asymmetry $\left(f=L_{i} L_{j}, \tilde{L}_{i} \tilde{L}_{j}\right)$ :

$$
\begin{aligned}
\epsilon_{-} & =\Delta L \frac{\sum_{f}\left[\Gamma\left(T_{-}^{*} \rightarrow f\right)-\Gamma\left(T_{-} \rightarrow \bar{f}\right)\right]}{\Gamma_{T_{-}^{*}}+\Gamma_{T_{-}}} \\
& =-\frac{1}{8 \pi} \frac{1}{M_{T} \Gamma} \frac{\left(M_{-}^{2}-M_{+}^{2}\right) \sum_{f} \operatorname{Im}\left(S_{-}^{f *} S_{+}^{f} \Pi_{+-}\right) c_{f}}{\left(M_{-}^{2}-M_{+}^{2}\right)^{2}+\Pi_{++}^{2}},
\end{aligned}
$$

where $\Delta L=2$ and the amplitudes $S^{f}$ can be read off from eq. (15) as follows: $S=2 h$ ( $h$ is the corresponding coupling in (5) for the scalar final states and $S=2 M_{T} h$ for the fermionic final states. Notice that the denominator involves not only the decays into leptons and sleptons, as the numerator does, but the total decay width $\Gamma_{T_{-}^{*}}=\Gamma_{T_{-}} \equiv \Gamma$ of any member of the $T_{-}$and $T_{-}^{*}$ triplets with

$$
\Gamma=\sum_{A} \Gamma_{0}^{A} c_{A}
$$

where $A=(a, \tilde{a})$ with $a=\left(L, \tilde{H}_{1}, \tilde{H}_{2}\right), \tilde{a}=\left(\tilde{L}, H_{1}, H_{2}\right)$, and the various partial decay widths into a pair of $a$ or $\tilde{a}$ at zero temperature are given by

$$
\begin{aligned}
\Gamma_{0}^{L} & =\Gamma_{0}^{\tilde{L}}=\frac{M_{T}}{32 \pi} \operatorname{Tr}\left(Y_{T} Y_{T}^{\dagger}\right), \\
\Gamma_{0}^{\tilde{H}_{i}} & =\Gamma_{0}^{H_{i}}=\frac{M_{T}}{32 \pi}\left|\lambda_{i}\right|^{2}, \quad i=1,2 .
\end{aligned}
$$

In eqs. (7) and (8) the $c$ factors are the contributions to the phase-space factors of the final states due to finitetemperature effects:

$$
\begin{aligned}
c_{a} & =\left(1-2 x_{a}\right) \sqrt{1-4 x_{a}}\left[1-n_{F}\left(E_{a}\right)\right]^{2}, \\
c_{\tilde{a}} & =\sqrt{1-4 x_{\tilde{a}}}\left[1+n_{B}\left(E_{\tilde{a}}\right)\right]^{2} .
\end{aligned}
$$

They reduce to 1 for $T=0$. Here we have defined:

$$
\begin{array}{cc}
E_{a(\tilde{a})}=\frac{m_{T}(T)}{2}, & x_{a(\tilde{a})} \equiv \frac{m_{a(\tilde{a})}^{2}(T)}{m_{T}^{2}(T)} \\
n_{F}(E)=\frac{1}{e^{E_{a} / T}-1}, & n_{B}(E)=\frac{1}{e^{E_{\tilde{a}} / T}+1},
\end{array}
$$

where the thermal masses read as (see e.g. 20])

$$
\begin{aligned}
m_{T}^{2}(T) & =M_{T}^{2}+\left(g_{2}^{2}+\frac{1}{2} g_{Y}^{2}\right) T^{2} \\
m_{\tilde{L}}^{2}(T) & =2 m_{L}^{2}(T)=m_{H_{1}}^{2}(T)=\left(\frac{3}{8} g_{2}^{2}+\frac{1}{8} g_{Y}^{2}\right) T^{2} \\
m_{H_{2}}^{2}(T) & =\left(\frac{3}{8} g_{2}^{2}+\frac{1}{8} g_{Y}^{2}+\frac{3}{4} y_{t}^{2}\right) T^{2}
\end{aligned}
$$

Here $g_{2}$ and $g_{Y}$ are the $S U(2)_{L}$ and $U(1)_{Y}$ gauge couplings, respectively, and $y_{t}$ is the top Yukawa coupling (to be renormalized at the appropriate high-energy scale).

In eq. (7) and in the following we keep only the lowestorder contribution in the soft terms, i.e. we use the approximation $\Gamma_{T_{-}}=\Gamma_{T_{+}}=\Gamma$ and $M_{+}=M_{-}=M_{T}$, whenever it concerns only higher-order corrections. Regarding the factors $\Pi_{ \pm \pm}$and $\Pi_{+-}$in eq. (7), the diagrammatic computations give:

$$
\begin{aligned}
& \Pi_{ \pm \pm}=M_{T}\left[\Gamma_{0}^{L}\left(R_{L}+R_{\tilde{L}}\right)+\sum_{i} \Gamma_{0}^{\tilde{H}_{i}}\left(R_{\tilde{H}_{i}}+R_{H_{i}}\right)\right] \\
& \Pi_{+-}=\Pi_{-+}^{*}=i \frac{M_{T}}{16 \pi} \operatorname{Im}\left[\operatorname{Tr}\left(A_{T} Y_{T}^{\dagger}\right) R_{\tilde{L}}(T)\right. \\
& \left.+\left(A_{1} \lambda_{1}^{*}\right) R_{H_{1}}(T)+\left(\lambda_{2} A_{2}^{*}\right) R_{H_{2}}(T)\right],
\end{aligned}
$$

where $R_{\tilde{a}}$ and $R_{a}\left(\tilde{a}=\tilde{L}, H_{1}, H_{2}, \quad a=L, \tilde{H}_{1}, \tilde{H}_{2}\right)$ are the finite temperature stimulated emission and Pauliblocking factors for the bosons and fermions in the loops, respectively [18]:

$$
R_{\tilde{a}}(T)=\sqrt{1-4 x_{\tilde{a}}}\left[1+2 n_{B}\left(E_{\tilde{a}}\right)+2 n_{B}^{2}\left(E_{\tilde{a}}\right)\right],
$$

while for the (cumbersome) expression of $R_{a}$ we refer the reader to eq. D1 in [18]. We recall that the $R$ factors are different from the $c$ factors in eqs. (11) and (12), as explained in [18]. Note also that only bosonic loops contribute to $\Pi_{+-}$and therefore the $\mathrm{CP}$-asymmetries are enhanced by the stimulated emission factors in $R_{a}$. As for the $L$-asymmetry $\epsilon_{+}$from the $T_{+}$decays, we have that $\epsilon_{+}=\epsilon_{-}$. Then by combining these contributions to the lepton asymmetry, we obtain:

$$
\epsilon=\epsilon_{-}+\epsilon_{+}=\kappa\left(c_{\tilde{L}}-c_{L}\right)
$$

where

$$
\kappa=\left(\frac{16 B M_{T} \operatorname{Im} \Pi_{+-}}{4 B^{2} M_{T}^{2}+\Pi_{++}^{2}}\right) \frac{\Gamma_{0}^{L}}{\Gamma} .
$$

Notice that a non-zero asymmetry is obtained because two important ingredients are present: 1) there are physical (unremovable) relative phases among the superpotential and related soft-trilinear couplings, 2) finitetemperature effects make the fermion and boson phasespaces different, $c_{L} \neq c_{\tilde{L}}$. Another important feature of our leptogenesis scenario is that it displays a resonance behaviour [21] for $2 B M_{T} \simeq \Pi_{++}$[see eq. [20]], which depends on the temperature.

From $\epsilon$ the total produced baryon asymmetry $n_{B} / s$ can now be calculated. Since the asymmetry is temperature dependent, the $n_{B} / s$ ratio cannot be expressed as the product of the CP-asymmetry times an efficiency factor which would be independent of this asymmetry. As most of the terms in $\epsilon$ are temperature dependent, all of the $\mathrm{CP}$-asymmetry has to be absorbed in the efficiency factor 
which is the result of (numerical) temperature integration of the Boltzmann equations. Here, instead, to have some physical insight onto the various effects, we rewrite the asymmetry as

$$
\epsilon=\epsilon_{0} C_{T}\left(c_{\tilde{L}}-c_{L}\right),
$$

with $\epsilon_{0}=\left.\kappa\right|_{T=0}$ and $C_{T}=\kappa /\left(\left.\kappa\right|_{T=0}\right)$. The three factors play distinct roles: $i) \epsilon_{0}$ exhibits the dependence of the asymmetry on the relevant interactions (5) at zero temperature, $i i)$ the factor $c_{\tilde{L}}-c_{L}$ encodes the crucial finite temperature effect leading to a non-vanishing asymmetry, $i i i$ ) the coefficient $C_{T}$ reflects residual (and minor) thermal effects and it is of order one. In this way, the temperature-dependent factors $C_{T}$ and $c_{\tilde{L}}-c_{L}$ can be absorbed in the efficiency parameter $\eta$ and finally the baryon asymmetry can be expressed (at the leading-order in the soft terms) as:

$$
\begin{aligned}
\frac{n_{B}}{s} & =-\left(\frac{24+4 n_{H}}{66+13 n_{H}}\right) \epsilon_{0} \eta 6 Y_{T}^{e q} \\
& =-3 \times 10^{-3} \epsilon_{0} \eta .
\end{aligned}
$$

The first factor takes into account the reprocessing of the $B-L$ asymmetry by sphaleron transitions, with the number of Higgs doublets $n_{H}=2$. The parameter $Y_{T}^{e q}=$ $45 \zeta(3) /\left(2 \pi^{4} g_{*}\right)$ is the equilibrium number density in units of entropy density ${ }^{5}$ for any component of the $T_{-}, T_{-}^{*}, T_{+}$ or $T_{+}^{*}$ triplets, and the factor 6 accounts for the 6 states in $T_{-}$and $T_{-}^{*}$ (or for the 6 states in $T_{+}$and $T_{+}^{*}$ ).

4. Results. For the sake of simplicity and discussion, let us make some reasonable assumptions to show that our mechanism can lead to the observed baryon asymmetry. First, we neglect the top Yukawa contribution ${ }^{6}$ in eq. (15), so that all phase space factors $c_{a}, c_{\tilde{a}}$, as well as $R_{a}, R_{\tilde{a}}$, are equal for all scalar states $\tilde{a}$ and for all fermion states $a$. Furthermore, we also neglect the temperature effect in $\Pi_{++}$, as the maximum value the asymmetry can take (at the resonance) is hardly affected by this approximation. In addition, by assuming 'universallike' relations for the trilinear couplings in eq. (3), i.e. $A_{T}=A Y_{T}, A_{1}=A \lambda_{1}, A_{2}=A \lambda_{2}$, the lepton asymmetry $\epsilon$ takes the simple form given by eq. (21) with

$$
\begin{gathered}
\epsilon_{0}=\left(\frac{16 \Gamma_{0} B}{4 B^{2}+\Gamma_{0}^{2}}\right) \frac{\operatorname{Im} A}{M_{T}} \frac{\Gamma_{0}^{L}}{\Gamma_{0}}, \\
C_{T}\left(c_{\tilde{L}}-c_{L}\right) \approx 2 R_{\tilde{L}} \Delta_{B F}
\end{gathered}
$$

\footnotetext{
5 In this supersymmetric extension of the standard model, the number of effective degrees of freedom is $g_{*}=251.25$.

6 This may appear quite a questionable assumption, as at high energy-scale $10^{7}-10^{10} \mathrm{GeV}$ the coupling $y_{t}$ is generically comparable (or even larger) than the gauge couplings $g_{2}, g_{1}$. So neglecting the $y_{t}$ contribution is merely motivated by simplicity and do not spoil our illustrative presentation.
}

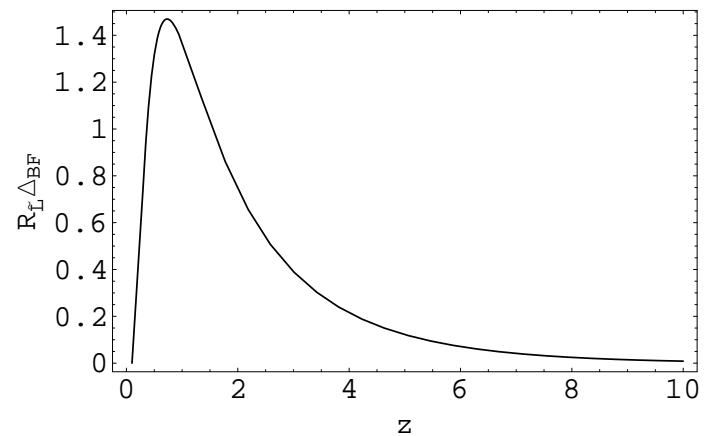

FIG. 2: The quantity $R_{\tilde{L}} \Delta_{B F}$, defined in 24, as a function of $z=M_{T} / T$.

where $\Gamma_{0}=\sum_{p} \Gamma_{0}^{p}$ is the total decay width $(p=L, \tilde{L}$, $\left.H_{1}, \tilde{H}_{1}, H_{2}, \tilde{H}_{2}\right)$ at zero-temperature [see eqs. (9), (10)] and [14]

$$
\Delta_{B F}=\frac{c_{\tilde{L}}-c_{L}}{c_{\tilde{L}}+c_{L}}
$$

In Fig. 2 the temperature dependent factor $R_{\tilde{L}} \Delta_{B F}$ is plotted as a function of $z=M_{T} / T$. For $T \gg M_{T}$ this factor and hence the asymmetry vanish because no scalar decay modes are kinematically available. For $T \sim M_{T}$ both the decays $T_{ \pm} \rightarrow \bar{L} \bar{L}, \tilde{L}^{*} \tilde{L}^{*}$ become possible and so $\epsilon$ is unsuppressed due to the finite-temperature effects. For $T \ll M_{T}, \epsilon$ vanishes because the asymmetries in $L$ and $\tilde{L}$ exactly cancel against each other $\left(\Delta_{B F}=0\right)$. The asymmetry attains its resonant maximum value for $2 B=\Gamma_{0}$ :

$$
\epsilon^{\max }=8 \frac{\operatorname{Im} A}{M_{T}} \frac{\Gamma_{0}^{L}}{\Gamma_{0}} R_{\tilde{L}} \Delta_{B F} .
$$

If, for example, we take $M_{T}=10^{8} \mathrm{GeV}, \operatorname{Im} A \sim 1 \mathrm{TeV}$ and $\Gamma_{0}^{L} \approx \Gamma$, from eq. (22) we see that the efficiency parameter needs to be $\sim \mathcal{O}\left(10^{-3}\right)$ in order to explain the observed $n_{B} / s \approx 8 \times 10^{-11}$. The efficiency $\eta$ is a function of $\Gamma$ and $M_{T}$ and, since the triplets have gauge couplings, $\eta$ is expected to be smaller than in the singlet leptogenesis case [9, 22]. Although we have not solved the Boltzmann equations, we believe that, based on the results obtained in [9, 22], $\eta \sim \mathcal{O}\left(10^{-3}\right)$ is realistic. For this estimate we use the efficiency factor given in Fig. 6 of Ref. [22] for the case of a fermion triplet, under the assumption that it does not differ much from the one in the case of a scalar triplet. ${ }^{7}$ This estimate takes also into account the results of Fig. 2 as well as the fact that

\footnotetext{
7 Gauge scatterings are expected to be essentially the same up to factors of order one. A more precise determination of the produced baryon asymmetry, including in particular the calculation of the efficiency factor for a scalar triplet, is beyond the scope of this letter and is left for a subsequent publication.
} 
$\Gamma_{0} / H\left(M_{T}\right)$ is bounded from below by the value $\sim 40$, and hence some washout (though moderate) is active due to the inverse decay processes. This bound on $\Gamma_{0} / H\left(M_{T}\right)$ is derived by minimizing the total width $\Gamma_{0}$, which can be more conveniently expressed in terms of the neutrino masses $m_{\alpha}, \alpha=1,2,3$ [see also eq. (2)], i.e.:

$$
\Gamma_{0}=\frac{M_{T}}{16 \pi}\left(\frac{M_{T}^{2}}{\lambda_{2}^{2} v_{2}^{4}}\left(\sum_{\alpha} m_{\alpha}^{2}\right)+\lambda_{1}^{2}+\lambda_{2}^{2}\right) .
$$

The minimum is achieved for $\lambda_{1}=0$ and for

$\lambda_{2}=\frac{\sqrt{M_{T} \sqrt{\sum_{\alpha} m_{\alpha}^{2}}}}{v_{2}} \sim 4 \times 10^{-4}\left(\frac{M_{T}}{10^{8} \mathrm{GeV}} \frac{m_{3}}{0.05 \mathrm{eV}}\right)^{1 / 2}$,

where the neutrino masses are inferred by the present data (and assuming hierarchical neutrino spectrum). So the least value of $\Gamma_{0}$ is:

$$
\Gamma_{0}^{m i n}=\frac{1}{8 \pi} \frac{M_{T}^{2} \sqrt{\sum m_{\alpha}^{2}}}{v_{2}^{2}} \sim 1 \mathrm{GeV}\left(\frac{M_{T}}{10^{8} \mathrm{GeV}}\right)^{2}
$$

and given that $H(T) \simeq 17 T^{2} / M_{P l}$, we get the aforesaid bound:

$$
\frac{\Gamma_{0}}{H\left(T=M_{T}\right)} \gtrsim 40 \times\left(\frac{m_{3}}{0.05 \mathrm{eV}}\right),
$$

which does not depend on $M_{T}$. Now let us discuss the range of $M_{T}$ for which successful leptogenesis can be achieved. By considering eq. (26) and our subsequent discussion, we can see that leptogenesis requires $M_{T}$ in the range $\sim 10^{3}-10^{9} \mathrm{GeV}$ with the parameter $\operatorname{Im}(A)$ in the range $(0.1-10) \mathrm{TeV}$. Higher values for $M_{T}$ would lead to a smaller baryon asymmetry because of the $1 / M_{T}$ suppression in eq. (26). Another important issue is the size of the $B$-parameter implied by the triplet leptogenesis. Consider the most favourable situation when the resonance is realized and taking the minimum total decay width of eq. (29), i.e. with $B=\frac{1}{2} \Gamma_{0}^{\min }$. Then for $M_{T}=10^{8} \mathrm{GeV}\left(10^{9} \mathrm{GeV}\right)$ we have $B \sim 1 \mathrm{GeV}$ $(100 \mathrm{GeV})$. Note that a value of $M_{T}$ as low as $1 \mathrm{TeV}$, which is not prevented by the efficiency factor as shown in Ref. [22], requires a very tiny value of $B$, of order $10^{-10} \mathrm{GeV}$, which would call for an explanation [23].

5. General remarks. For the sake of completeness, we would like to comment on some additional related issues. First, in the supersymmetric case with gravitino mass close to the electroweak scale, avoiding the gravitino-overclosure of the Universe implies an upper bound $T_{R H}<10^{8} \mathrm{GeV}$ on the reheating temperature 24]. This is a potential problem in the singlet seesaw leptogenesis models which (assuming hierarchical singlet neutrinos) require $M_{N_{1}}>2.4 \times 10^{9} \mathrm{GeV}[18,25]$ (see, however, ref. 22] ). This bound is not in conflict with our triplet leptogenesis mechanism which can accommodate values of $M_{T}$ below $10^{8} \mathrm{GeV}$.
Another related issue concerns the presence of such $S U(2)_{L}$ triplets at intermediate energy, below the grand unification scale $M_{G}$, which would spoil the gauge coupling unification. The latter can be maintained by embedding our scenario in a larger gauge theory, such as, for example, the $S U(5)$ model. In this case the triplets $T(\bar{T})$ fit into the $15(\overline{15})$ representation, together with extra states $S(\bar{S})$ and $Z(\bar{Z})$ which decompose according to $\left.\left.S U(3)_{c} \times S U(2)_{L} \times U\right) 1\right)_{Y}$ as $S \sim(6,1,-2 / 3), Z \sim$ $(3,2,1 / 6)$. In the $S U(5)$ broken phase, we have the couplings involving $S$ and $Z$, namely $Y_{S} S d^{c} d^{c}, Y_{Z} Z d^{c} L\left(d^{c}\right.$ are the $S U(2)_{L}$ singlet down quarks, see [6] for more details), and those in eq. (11) involving the triplets $T, \bar{T}$. The interactions with the $S U(3)_{c}$-coloured Higgs partners $t_{1}$ and $t_{2}$, namely $S t_{1} t_{1}, Z t_{1} H_{1}\left(\bar{S} t_{2} t_{2}, \bar{Z} t_{2} H_{2}\right)$, do not appear as $t_{1}$ and $t_{2}$ are decoupled at $M_{G}$ to prevent too rapid a proton decay. Therefore, the interactions of $S$ and $Z$ with the matter fields below $M_{G}$ are $B-L$ conserving (while those of the triplets $T$ are not) and so they cannot play any role in the generation of the baryon asymmetry. Though at $M_{G}$ the above interactions with the coloured Higgs states $t_{1}, t_{2}$ violate $B-L$, it is likely that any excess of $B-L$ number produced by these interactions is washed-out by the $B-L$ violating interactions of the triplets $T, \bar{T}$. The final produced asymmetry is therefore the one given only by the decays of the $S U(2)_{L}$ triplets.

The main attractiveness of the minimal supersymmetric type-II scenario is that the high-energy flavour structure can be univocally determined from the low-energy neutrino mass matrix. We also recall another appealing feature related to the above one. Namely, the Yukawa matrix $Y_{T}$ can induce radiative corrections in the (softbreaking) mass matrix $m_{\tilde{L}}$ of the sleptons $\tilde{L}$ and, as a result, lepton flavour violating non-diagonal entries are generated, even if, for example, $m_{\tilde{L}}$ is flavour-blind at $M_{G}$. Due to the direct connection between $Y_{T}$ and the neutrino mass matrix, the flavour structure of the mass matrix $m_{\tilde{L}}$ can be determined in terms of the neutrino parameters and so correlations among lepton flavour violating processes involving different families can be predicted [ $[$ ] . The leptogenesis scenario we have presented depends not only on superpotential couplings as the neutrino masses do, but also on (several) soft-breaking parameters, whose determination would require (and certainly deserve) a proper phenomenological study to point up some correlations with other observables. Testing our leptogenesis scenario appears to be as involved as other high-scale realizations of leptogenesis.

6. Conclusions In this paper we have shown that the minimal supersymmetric seesaw scenario with a single pair of triplets can generate successful leptogenesis through the $L$-violating decays of these states. The necessary CP-asymmetry arises from the interference between supersymmetric and soft-breaking terms and by 
a resonant-enhancement due to the 'soft' mass splitting of the triplets $T$ and $\bar{T}$. In this scenario the mass of the triplet states should be in the range $\left(10^{3}-10^{9}\right) \mathrm{GeV}$ depending on the size of the soft-supersymmetry breaking parameters.

Acknowledgment. We thank M. Pietroni, A. Strumia, S. Vempati and J. Valle for useful discussions. The work of G. D. was partially supported by IHP-RTN, EC contract No. HPRN-CT-2002-00311 (EURIDICE), of T. H. by the European Union (EU) Marie Curie HPMFCT-01765 and HPRN-CT-2000-00152 (Susy in the Early Universe) contracts, of M. R. by the ESF Grants 5135 and 5935, by the EC MC contract MERG-CT-2003-503626, and by the Ministry of Education and Research of the Republic of Estonia, of A. R. by the EU HPRN-CT-200000148 (Across the Energy Frontier) and HPRN-CT-200000149 (Collider Physics) contracts.

[1] M. H. Ahn et al. [K2K Collab.], Phys. Rev. Lett. 90, 041801 (2003) arXiv:hep-ex/0212007; K. Eguchi et al. [KamLAND Collab.], Phys. Rev. Lett. 90, 021802 (2003) arXiv:hep-ex/0212021; S. N. Ahmed et al. [SNO Collab.], arXiv:nucl-ex/0309004 M. Ishitsuka [SuperKamiokande Collaboration], arXiv:hep-ex/0406076

[2] P. Minkowski, Phys. Lett. B 67 (1977) 421; M. GellMann, P. Ramond and R. Slansky, Proceedings of the Supergravity Stony Brook Workshop, New York, 1979, eds. P. Van Nieuwenhuizen and D. Freedman (NorthHolland, Amsterdam); T. Yanagida, Proceedings of the Workshop on Unified Theories and Baryon Number in the Universe, Tsukuba, Japan 1979 (eds. A. Sawada and A. Sugamoto, KEK Report No. 79-18, Tsukuba); S.L. Glashow, in Quarks and Leptons, Cargèse, eds. M. Lévy et al., (Plenum, 1980, New-York), p. 707; T. Yanagida, in Proceedings of the Workshop on the Unified Theory and the Baryon Number in the Universe, edited by O. Sawada and A. Sugamoto (KEK Report No. 79-18, Tsukuba, 1979), p. 95; R.N. Mohapatra and G. Senjanović, Phys. Rev. Lett. 44, (1980) 912.

[3] J. Schechter and J. W. F. Valle, Phys. Rev. D22 (1980) 2227; T. P. Cheng and L. F. Li, Phys. Rev. D22 (1980) 2860.

[4] M. Magg and C. Wetterich, Phys. Lett. B94 (1980) 61; C. Wetterich, Nucl. Phys. B187 (1981) 343; G. Lazarides, Q. Shafi and C. Wetterich, Nucl Phys. B181 (1981) 287; R.N. Mohapatra and G. Senjanović, Phys. Rev. D23 (1981) 165.

[5] P. H. Frampton, S. L. Glashow and T. Yanagida, Phys. Lett. B548 (2002) 119 arXiv:hep-ph/0208157. M. Raidal and A. Strumia, Phys. Lett. B553 (2003)
72 arXiv:hep-ph/0210021; R. Barbieri, T. Hambye and A. Romanino, JHEP 0303 (2003) 017 arXiv:hep-ph/0302118.

[6] A. Rossi, Phys. Rev. D $66 \quad$ (2002) 075003 arXiv:hep-ph/0207006.

[7] M. Fukugita and T. Yanagida, Phys. Lett. B 174 (1986) 45.

[8] E. Ma and U. Sarkar, Phys. Rev. Lett. 80 (1998) 5716.

[9] T. Hambye, E. Ma, U. Sarkar, Nucl. Phys. B602 (2001) 23 hep-ph/0011192.

[10] P. O'Donnell and U. Sarkar, Phys. Rev. D49 (1994) 2118; G. Lazarides and Q. Shafi, Phys. Rev. D58 (1998) 071702.

[11] T. Hambye and G. Senjanovic, Phys. Lett. B582 (2004) 73.

[12] W. Rodejohann, hep-ph/0403236 P. Gu and X. Bi, hep-ph/0405092 S. Antusch and S. King, hep-ph/0405093 W. Guo, hep-ph/0406268

[13] Y. Grossman, T. Kashti, Y. Nir and E. Roulet, Phys. Rev. Lett. 91 (2003) 251801 arXiv:hep-ph/0307081.

[14] G. D'Ambrosio, G. F. Giudice and M. Raidal, Phys. Lett. B575 (2003) 75 arXiv:hep-ph/0308031.

[15] L. Boubekeur, T. Hambye and G. Senjanovic, hep-ph/0404038

[16] Y. Grossman, T. Kashti, Y. Nir and E. Roulet, hep-ph/0407063

[17] L. Boubekeur, arXiv:hep-ph/0208003.

[18] G. F. Giudice, A. Notari, M. Raidal, A. Riotto and A. Strumia, Nucl. Phys. B685 (2004) 89 arXiv:hep-ph/0310123.

[19] A. Pilaftsis, Phys. Rev. D56 (1997) 5431, arXiv:hep-ph/9707235.

[20] D. Comelli and J. R. Espinosa, Phys. Rev. D55 (1997) 6253 arXiv:hep-ph/9606438.

[21] M. Flanz, E.A. Paschos and U. Sarkar, Phys. Lett. B345 (1995) 248; L. Covi, E. Roulet and F. Vissani, Phys. Lett. B 384 (1996) 169 arXiv:hep-ph/9605319; M. Flanz, E.A. Paschos, U. Sarkar and J. Weiss, Phys. Lett. B389 (1996) 693; A. Pilaftsis, Nucl. Phys. B504 (1997) 61; J. R. Ellis, M. Raidal and T. Yanagida, Phys. Lett. B 546 (2002) 228 arXiv:hep-ph/0206300; A. Pilaftsis and J. Underwood, hep-ph/0309342 T. Hambye, J. MarchRussell and S. West, hep-ph/0403183.

[22] T. Hambye, Y. Lin, A. Notari, M. Papucci and A. Strumia, arXiv:hep-ph/0312203

[23] For such a possible explanation see e.g. in another context: J. March-Russell and S. West, Phys. Lett. B593 (2004) 181.

[24] M. Y. Khlopov and A. D. Linde, Phys. Lett. B 138 (1984) 265; J. R. Ellis, D. V. Nanopoulos and S. Sarkar, Nucl. Phys. B259 (1985) 175; J. R. Ellis, D. V. Nanopoulos, K. A. Olive and S. J. Rey, Astropart. Phys. 4 (1996) 371; M. Kawasaki and T. Moroi, Prog. Theor. Phys. 93 (1995) 879.

[25] S. Davidson and A. Ibarra, Phys. Lett. B535 (2002) 25 arXiv:hep-ph/0202239. 